\title{
Abbruch, Umbruch, Aufbruch: Programme des sächsischen Lokalfernsehens in der Nachwendezeit 1990-1995
}

\author{
Judith Kretzschmar \& Rüdiger Steinmetz
}

Keywords: Transformation, Sattelzeit 1990-1995, Lokalfernsehen, Identität, Ostdeutschland, Vereinigungsprozess

\section{Abstract}

Von 1989/90 bis 1995 kam es in Deutschland zu einem Zusammenprall der Kulturen West versus Ost, bei dem die Macht ungleich verteilt war. Die etwa 40 sächsischen Lokalfernsehprogramme aus dieser Zeit stellen - mit dem »Blick von unten «- einzigartige Quellen für das kulturelle Gedächtnis eines der wichtigsten historischen Einschnitte im 20. Jahrhundert dar. Eine Untersuchung der Programme und deren heutige Wiederaufführung tragen zur Revision etablierter Darstellungen, Positionen und Beurteilungen dieser Zeit bei. In diesem Beitrag wird auf Zwischenergebnisse zweier Untersuchungskategorien eingegangen: einerseits auf Umbrüche im Arbeitsleben und deren - als teilweise bis heute fortdauernde Ängste-erlebte Auswirkungen sowie andererseits auf die Begegnung mit Westdeutschen. Aus den im Projekt realisierten Wiederaufführungen von Lokal-TV-Beiträgen in ganz Sachsen lassen sich heute bei den Zuschauer*innen Lebenserfahrungen sowie verschüttete, verdrängte beziehungsweise (nur) gelernte, erworbene Kontexte und Gefühle dieser Sattelzeit re-aktualisieren. Außerdem können dadurch Erklärungen für die Wurzeln gegenwärtiger sozialer Probleme und politischer Diskurse im vereinigten Deutschland gegeben werden.

Judith Kretzschmar \& Rüdiger Steinmetz: Abbruch, Umbruch, Aufbruch: Programme des sächsischen Lokalfernsehens in der Nachwendezeit 19901995. In: Nils S. Borchers, Selma Güney, Uwe Krüger und Kerem Scham- 
berger (Hrsg.): Transformation der Medien - Medien der Transformation. Verhandlungen des Netzwerks Kritische Kommunikationswissenschaft. Frankfurt am Main: Westend 2021. DOI: https://doi.org/10.53291/THLT5265.

Dr. Judith Kretzschmar I Leipziger Institut für Heimat- und Transformationsforschung (LIHT) I jkretz@uni-leipzig.de

Prof. Dr. Rüdiger Steinmetz I Leipziger Institut für Heimat- und Transformationsforschung (LIHT) I rstein@uni-leipzig.de

\section{Einführung}

Im Zeitraum der Niederschrift dieses Beitrags, im Oktober 2020, erreichten uns ständig Interviewanfragen von »Qualitätsmedien«, die Expertise aus unserem vor mehr als zehn Jahren abgeschlossenen und breit veröffentlichten DFG-Forschungsprojekt über die Programmgeschichte des DDR-Fernsehens wollten: ${ }^{1}$ Zur Aktuellen Kamera, zum Fernsehballett, zur ostalgischen Unterhaltungsorientierung des MDR, zum medizinischen Beratungsformat Visite - und wir erwarten noch Fragen zu Karl-Eduard von Schnitzler (Der Schwarze Kanal), also »Sudel-Ede«, »Pittiplatsch« und zum Sandmann mit dem Spitzbart. Weil das Internet nichts vergisst, finden einfallslose, klischee-orientierte und an wiederkehrender Jubiläumsberichterstattung interessierte Journalist*innen von heute immer wieder dieselben Themen. Ihre eigenen Recherchen ergeben nur wenig zu medialen Vereinigungsbilanzen, den neuen biographischen Selbstreflexionen der »Wende- $\ll^{2}$ und der »Nachwende-Generation« und schon gar nichts zu neuen medialen Quellen, die im Folgenden dargestellt werden.

1 Vgl. Steinmetz und Viehoff (2008). Judith Kretzschmar war eine der Ko-Autor*innen. Aus diesem DFG-Projekt gingen mehr als 30 Publikationen in der Reihe »MAZ. Materialien, Analysen, Zeitzeugen« im Universitätsverlag Leipzig hervor.

2 Die deutsche Geschichte des 20. Jahrhunderts ist voller Brüche, beispielsweise in den Jahren 1918, 1933, 1945 oder 1989. Doch nur für den gewaltigen Umbruch in der DDR bürgerte sich der belanglose Begriff »Wende « ein. Zurückzuführen ist dies auf Egon Krenz, den Nachfolger Erich Honeckers. Dieser sprach in seiner Antrittsrede am 18. Oktober 1989 in Anlehnung an die sowjetische Perestroika von einer nötigen »Wende« durch die SED. Allerdings ist »Wende« kein überzeugender Terminus für diesen fundamentalen Systemwechsel, da er das Ausmaß der Veränderungen überhaupt nicht abdeckt (vgl. Jesse o. J.; Richter 2007a; 2007b; Eckert 2007). 
Dieser Beitrag präsentiert Zwischenergebnisse des noch laufenden Forschungsprojekts »Heimat heute - oder: Jeder hat sein Nest im Kopf «. ${ }^{3}$ In dessen Mittelpunkt steht die Analyse eines einzigartigen Bestandes an audiovisuellen Quellen, die das kulturelle Gedächtnis der Menschen in Ostdeutschland in der Sattelzeit ${ }^{4}$ zwischen 1989/90 und 1995 bewahren und den Vereinigungsprozess abbilden: die Programme von bis zu 40 lokalen Fernsehsendern in Sachsen. Sie sind bis heute weder von der Forschung noch von der Politik, den staatlichen Archiven oder den öffentlich-rechtlichen Qualitätsmedien in größerem Umfang beachtet worden.

Zum ersten Mal in der Geschichte wird mit diesem singulären Fundus an lokalen Fernsehprogrammen ein wichtiger deutscher und europäischer Transformationsprozess kontinuierlich durch bewegte Bilder festgehalten, und zwar in einem Bottom-up-Produktionsprozess sich selbst medial professionalisierender Bürger*innen und nicht in einem staatlichen Top-down-Verlautbarungsprozess einer Machtelite. Wir orientieren uns an den von Ilko-Sascha Kowalczuk $(2019,213-214)$ formulierten Desideraten: „Wir brauchen eine kritische Auseinandersetzung mit den sozialen, wirtschaftlichen und kulturellen Ursachen und Folgen der Transformation. In dieser Aufarbeitung müssen alle Erfahrungsräume Platz finden.«

Von folgender Annahme wird dabei ausgegangen: Analysen der Programme der lokalen Fernsehsender in den neuen Bundesländern am Beispiel Sachsens (1990 bis 1995) sowie die re-aktualisierte Erfahrung aus heutiger Sicht (Fokusgruppen) können verschüttete, verdrängte beziehungsweise (nur) gelernte, erworbene Kontexte und Gefühle aufdecken sowie Erklärungen für die Wurzeln gegenwärtiger sozialer und politischer Diskurse im vereinigten Deutschland bieten.

Daraus wurden folgende Forschungsfragen entwickelt:

1. Inwieweit lassen sich aus der Begegnung mit diesen historischen Programmen Lebenserfahrungen sowie verschüttete beziehungsweise verdrängte Kontexte und Gefühle dieser Sattelzeit bei heutigen Seher*innen re-aktualisieren?

2. Inwieweit können dadurch gegenwärtige soziale und politische

3 Leipziger Institut für Heimat- und Transformationsforschung (LIHT), https:// www.heimat-und-transformation.de/en/.

4 Der Begriff der »Sattelzeit« wird in diesem Beitrag in Anlehnung an Reinhart Koselleck in seinen Geschichtlichen Grundbegriffen verwendet, der ihn in Bezug auf eine idealtypische Beschreibung des Übergangs von der alteuropäischen zur modernen Geschichtsauffassung, also den Umbruch vom Ende des 18. zum 19. Jahrhundert prägte (vgl. Koselleck 1972, XIII-XXIII). 
Diskurse im vereinigten Deutschland auf ihre Ursprünge zurückgeführt und erklärt werden?

\section{Die Große Transformation und ihr audiovisuelles Gedächtnis}

\subsection{Die Neue Große Transformation}

Die Jahre 1989 und $1990^{5}$ stellen neben 1918 und 1945 die dritte große Zäsur im 20. Jahrhundert dar. Sie markieren den Beginn einer tiefgreifend und zeitrafferartig vor sich gehenden Transformation des politischen, ökonomischen wie gesellschaftlichen Systems und damit des Alltags von Millionen Menschen. Dies ist von Ther (2019) in Anlehnung an den Wirtschaftshistoriker Karl Polanyi (2019 [1944]) die »Neue Große Transformation « genannt worden. »Große Transformation« bedeutet dabei eine tiefgreifende Umformung ohne revolutionäre Prozesse, also eine gewaltlose Veränderung verfestigter Formen in diversen Subsystemen (Staatlichkeit, Wirtschaft, Kultur und Gesellschaft) sowie grundlegende Strukturbrüche und -wandlungen (vgl. Buchholz 1968).

Dieser Prozess umspannt mehrere übergreifende Themenkomplexe, die der Forschung als analytische Kategorien zugrunde gelegt werden, nämlich der politische Systemwandel mit der Etablierung und Konsolidierung von Demokratie, Partei- und Verwaltungsstrukturen; dann der ökonomische Umbau: Kapitalismus statt Staatssozialismus, der neoliberale Umbau des Wirtschaftssystems durch Abwicklung oder Privatisierung bisher staatlicher Betriebe, unter anderem mit (zunächst) hoher Arbeitslosigkeit und späterer Prosperität als eine Folge dieser Entwicklungen (vgl. Ther 2016, 26, 28; Ther 2019, 166; Milev 2020, $23-40)^{6}$

5 1989: Fall der Mauer und anderer militärisch hoch bewehrter Grenzen in Mitteleuropa; 1990: Vereinigung Deutschlands, zunehmende Autonomie mittel-osteuropäischer Staaten, allmählicher Zerfall der Sowjetunion, Ende des Kalten Krieges.

6 Internationale Kontexte wie der Zusammenbruch des Staatssozialismus und seine Rückwirkungen auf die Europäische Gemeinschaft und die Europäische Union sowie auf die weltweiten politischen Koordinaten sind von hoher Wichtigkeit, werden aber in dem hier dargestellten Projekt noch nicht berücksichtigt. 
Diese Große Transformation war und ist nicht auf die DDR und die anderen postsozialistischen Staaten beschränkt, sondern umfasste, wie Philipp Ther (2019, 91-95) es bezeichnet, in einem ko-transformativen Prozess die »alte Bundesrepublik« und - mindestens - die Europäische Gemeinschaft sowie (ab 1992) die Europäische Union. Aus ihrer Geschwindigkeit ergaben sich aber folgende Besonderheiten der deutschen Transformation im Unterschied zu anderen postsozialistischen Staaten:

1. Der paritätische Umtauschkurs von einer D-Mark für eine DDRMark (statt real 1:4) ${ }^{7}$ bei der Währungsunion am 1. Juli 1990 bedeutete für die ostdeutsche Wirtschaft eine vierfache Verteuerung ihrer Ausfuhren gegenüber 1988. Er ermöglichte Produktionsverlagerungen nach Mittel- und Osteuropa an Ostdeutschland vorbei, wovon zum Beispiel Tschechien profitierte (vgl. Ther 2019, 79).

2. Ein »Zweiter Schock für die ostdeutsche Wirtschaft [war] die rasche Liberalisierung des Außenhandels« (ebd., 80). Wegen der EG fielen »sämtliche Handelsschranken nach Westeuropa - diesem Konkurrenzdruck war die ostdeutsche Wirtschaft nicht gewachsen « (ebd.).

3. Zwischen 1990 und 1992 wurden im Rahmen der »radikalen Privatisierung « (ebd.) mehr als 10000 ostdeutsche Betriebe verkauft. Dies führte zu einem drastischen Verfall der Preise für die Betriebe und zu einem Treuhand-Verlust von 270 Milliarden DM statt erwarteter Gewinne von 600 Milliarden DM (ebd., 80-81). Nur jeder vierte Arbeitsplatz blieb erhalten, was einen »Strukturbruch« vor allem für Städte mittlerer Größe bedeutete und einen »Bruch im Leben von Millionen von Menschen, die arbeitslos wurden, umschulen mussten, oft trotzdem keine dauerhafte Stelle fanden und durch Frühverrentung aufs Altenteil geschoben wurden« (ebd.).

4. Der Austausch der Eliten in Ostdeutschland war im Ergebnis radikaler als die Entnazifizierung in Westdeutschland nach 1945 (vgl. Weber 2020, 1129).

7 Der Umtauschkurs war gestaffelt und variierte je nach Alter: Ein Verhältnis 1:1 galt bei Bürger*innen ab 60 Jahren für bis zu 6000, zwischen 14 und 59 Jahren für bis zu 4000 und bei Kindern bis 14 Jahren für bis zu 2000 DDR-Mark, außerdem für regelmäßige Zahlungen wie Löhne, Gehälter, Stipendien, Renten, Mieten und Pachten. Höhere Sparguthaben und Schulden wurden 2:1 umgetauscht. Der Durchschnitt lag bei 1,8:1 (vgl. Neubert 2008, 386-387). 
Jeder Ostdeutsche hatte sein Leben »in einem Maße umstellen müssen, als wäre er in ein anderes Land gezogen. Fast alle wechselten den Arbeitsplatz, mit Phasen der bis dahin unbekannten Arbeitslosigkeit. Umlernen mussten alle, am Arbeitsplatz, in der Politik, in der Verwaltung, im privaten Bereich« (Schröder 2007, 13).

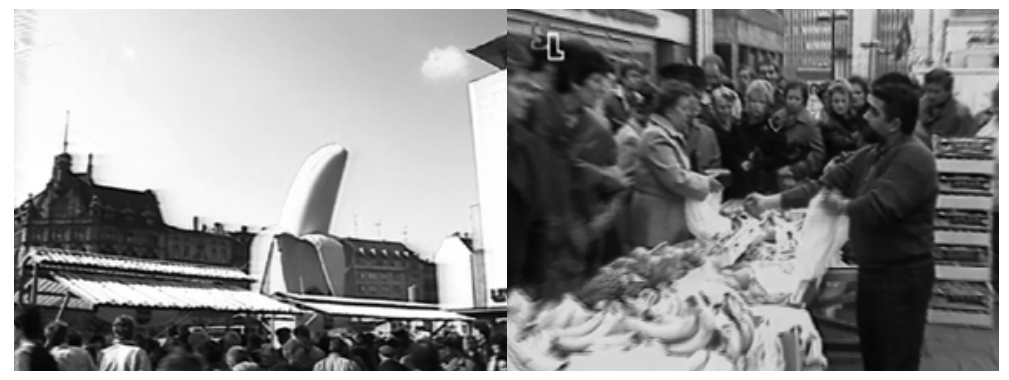

Abb. 1 und 2: März 1990 auf dem Leipziger Marktplatz: Marktschreier und eine überdimensionale Banane symbolisieren die westliche Übernahme öst-

licher Märkte. (C) Stadtfernsehen / Studio Leipzig, März 1990.

Die Große Transformation nahm allerdings nicht erst am 9. November 1989 oder am 3. Oktober 1990 ihren Anfang, und sie mündete nicht irgendwann am Ende der 1990er Jahre in eine vollendete Vereinigung, sondern hatte ihren Vorlauf in den 1980ern: Niedergang der DDR-Ökonomie, mediale Öffnung nach Westen, Entstehen und Erstarken einer oppositionellen Zivilgesellschaft, die sich die seit Anfang der 1980er Jahre aufkommenden, modernen elektronischen Medien zunutze machte (Satellitenfernsehen, Kabelnetzwerke, Antennengemeinschaften, Videokameras). Die Große Transformation ist ein bis heute andauernder, Generationen überspannender Prozess.

\subsection{Eingrenzungen und Ausprägungen des Projekts}

Das hier vorgestellte Forschungsprojekt konzentriert sich auf die Sattelzeit des Übergangs von einem staatssozialistischen in ein demokratisches und kapitalistisches System, auf die erste Phase der Transformation in ein gemeinsames Deutschland 1990 bis 1995. Diese ist unter anderem gekennzeichnet durch politische und mediale Maßnahmenund Ereignisbündel, nämlich »die Delegitimierung und Abschaffung der sozialistischen Staatsmacht in der DDR, [den] kurzzeitige[n] Ver- 
such des Aufbaus eines pluralistischen politischen Systems in der DDR, [den] zur Beendigung der deutschen Zweistaatlichkeit hinführende[n] Prozess sowie die politisch-administrative Übergangsperiode für das ostdeutsche Siedlungsgebiet« (Pasternak 1998, 21).

In diesem Zeitraum, Anfang 1995, endeten auch die ökonomischen Maßnahmen zur Übergangsfinanzierung der deutschen Einheit, und die öffentliche Finanzwirtschaft des vereinigten Deutschlands wurde weitgehend von Sonder- und Ausnahmeregelungen befreit (vgl. Wollmann 1997, 74; vgl. Einigungsvertrag 1990). Zwei weitere Gründe für den Zeitrahmen 1990 bis 1995 sind technischer Natur und von dem bisher Genannten weitgehend unabhängig: Zum einen datieren die audiovisuellen Quellen, die frühesten analogen Videobänder der ersten Gründungswelle der Lokalfernsehveranstalter, genau auf den Zeitraum von 1990 bis 1995 und sind von technischem Verfall bedroht. Zum anderen traf die Entfaltung gleich mehrerer für das Entstehen von Lokalfernsehen zentraler technologischer Entwicklungen mit den oben angeführten Entwicklungen zusammen, quasi als Conditiones sine qua non: tragbare Videokameras (Fernsehen für Jedermann) sowie Glasfaser- und Kupfer-Kabelnetzwerke.

Seit einigen Jahren ist, teilweise in einer jüngeren Generation, teilweise auch unter den ehemaligen Akteur*innen, ein Diskurs entstanden, der etliche der damaligen politischen und ökonomischen Entscheidungen kritisch daraufhin hinterfragt, ob sie tatsächlich so »alternativlos« waren, wie es vor 25 und 30 Jahren erschien. Jörg Schweinitz, Hilde Hoffmann und Tobias Ebbrecht postulierten bereits 2009 in Bezug auf das »abgeschlossene Sammelgebiet « DDR eine abgeklärte Haltung und ein nüchternes wissenschaftliches Urteil auf dem Feld der Transformationsforschung, um sich gar nicht erst in die Kämpfe der ehemaligen Protagonist*innen einzumischen (vgl. Schweinitz et al. 2009, 16-17). ${ }^{8}$ Hieraus leiten wir drei theoretisch-methodologische Momente ab:

8 Persönliche Anmerkungen dazu: Wir beide, als Autor*innen dieses Beitrags und als Forscher*innen heute, 30 Jahre nach diesem weitreichenden historischen Ereignis, versuchen einen solchen »frischen Blick« in der Forschung selbst einzunehmen. Den Fall der Mauer und die folgende Sattelperiode haben wir als reflektierende Erwachsene durchlebt: Judith Kretzschmar, die ihre prägenden politischen, kulturellen, sozialen und medialen Erfahrungen in der DDR gemacht hat, und Rüdiger Steinmetz in der Bundesrepublik Deutschland. Außerdem hat unser Thema seine Wurzeln in den Zeiten des Kalten Krieges zweier deutscher 
1. Heute stimmt es umso mehr, dass sich, frei von den Kämpfen der »heißen« Transformationszeit, eine abgewogenere Analyse anstellen lässt. Dies kann und muss (auch) eine jüngere Forscher*innengeneration leisten, gemeinsam mit Zeitzeugen und auf der Basis neuer, eben auch audiovisueller Quellen.

2. Vieles von dem, was heute aufbricht und sich in Wahlergebnissen niederschlägt, wurde vor 25 bis 30 Jahren unterschätzt oder falsch bewertet, in der Hektik des Umbruchs missachtet, liegengelassen, unter den Teppich gekehrt und überdeckt durch allzu viele, gleichzeitig auf Politik wie auf Bürger*innen einstürzende Herausforderungen, Verheißungen und Möglichkeiten. Heute stellen sich die damals unbeantworteten, verdrängten Fragen neu.

3. Gleichzeitig wirft die heutige Perspektive ein Licht auf die geronnenen Erfahrungen, Gefühle, Hoffnungen und Enttäuschungen, die in diesen frühen lokalen Fernsehprogrammen zu finden sind. Der Soziologe Michael Hofmann $(2016,7)$ betont: »Wir können heute noch gar nicht ermessen, wie wichtig und wertvoll die in der Transformation gewonnenen Lebenserfahrungen für die nachfolgenden Generationen sein werden.«

\subsection{Lokalfernsehen als Speicher audiovisuellen, kulturellen Gedächtnisses}

Spätestens seit Anfang der 1970er Jahre versteht man die Programme und Inhalte audiovisueller Massenmedien auch wissenschaftlich als geronnene Erinnerungen ihrer jeweiligen Zeit, als Bewegungen des "Zeitgeists « und als historische Quellen (vgl. Albrecht 1969; McCreary 1972). Auf der Produktebene werden die bewegten Bilder von Film und Fernsehen in ihrer synergetischen Einheit aus Ton, Montage und anderen untrennbaren ästhetischen Komponenten als zu analysierende historische und kulturelle Quellen immer mehr anerkannt und durch die Analyse der Bedingungen ihrer Produktion und Rezeption kontextualisiert. In modernen Gesellschaften sind die jeweiligen Mediensysteme auf (inter-)nationaler, regionaler und lokaler Ebene als komplexe, selbstreferentielle Kommunikationssysteme anzusehen, die eine »Sprache sprechen«. Auf der anderen Seite basieren die Inhalte dieser Pro-

Staaten, die jeweils in einen der gegnerischen Blöcke integriert waren und unsere Lebens- und Denkweisen beeinflusst haben. 
gramme auf lokalen und (sub-)regionalen Grundlagen; sie haben spezifische Wurzeln in Kultur und Geschichte. Diese klassischen Medien drücken einerseits gesellschaftliche Erfahrungen, Ideen und individuelle Phantasien aus und formen sie andererseits.

Massenmedien sind 》Systeme der Beobachtung sowie der Selbstbeobachtung der Gesellschaft« (vgl. Gerhards 1994) und sie sorgen für die Integration in und die Teilhabe von Menschen an der Gesellschaft. Sie vermitteln und übersetzen durch ihre audiovisuelle Repräsentation und Distribution zwischen der individuellen und der soziokulturellen Dimension des kollektiven Gedächtnisses (vgl. Erll 2017, 135). Die Kulturanthropologin Aleida Assmann (2018) untersuchte in ihrem Werk Der lange Schatten der Vergangenheit die Erinnerungskultur einschließlich der Rolle der Medien darin. Medien wie fiktionale und dokumentarische Filme oder im hier diskutierten Fall lokale Fernsehprogramme bilden Quellen lebendiger Erinnerungen, die diese in ein kulturelles Gedächtnis überführen:

Die Massenmedien schaffen wichtige Impulse und Auslöserreize für das kulturelle Gedächtnis, ohne selbst eines zu produzieren. Sie sind unentbehrlich, um es zu aktivieren, doch es bedarf anderer Instanzen, um es zu bilden. Der größte Teil der Medienangebote geht unwiederbringlich verloren; ein kleiner Teil landet im kulturellen Speichergedächtnis, ein winziger Ausschnitt davon wird Bestandteil des kulturellen Funktionsgedächtnisses (Assmann 2018, 242). ${ }^{9}$

Assmann unterscheidet grundsätzlich zwischen einem »inkarnierten Erlebnisgedächtnis« der Zeitgenoss*innen und einem »exkarnierten Mediengedächtnis« der Gesellschaft (Assmann 2018, 210-213), die sich gegenseitig beeinflussten und beeinflussen. Hinzugefügt sei: Im Laufe der Zeit, etwa bei Neuinterpretationen und der Wiederverwendung in verschiedenen Bildungskontexten, aber auch bei Neukompila-

9 Das »Speichergedächtnis« ist laut Assmann $(2018,55)$ der »Fundus und Hintergrund für latente Erinnerungen, die ihre Stunde hinter sich oder noch vor sich haben«, weil sie eben, wie es in dem hier vorgestellten Projekt geschieht, dem Vergessen durch Re-Aktualisierung entrissen und dem aktiven Erinnern im Funktionsgedächtnis zugeführt werden. »Im Funktionsgedächtnis herrscht notorischer Platzmangel. Was dort hineingelangt - vom Kanon biblischer Texte bis zum Kanon der Klassiker - ist durch rigorose Verfahren der Auswahl hindurchgegangen. [...] Was einen Platz im Funktionsgedächtnis einer Gesellschaft hat, hat Anspruch auf immer neue Aufführung, Ausstellung, Lektüre, Deutung, Auseinandersetzung.« (ebd., 56) 
tionen und Interpretationen in neuen Fernsehsendungen, hat dieses exkarnierte Mediengedächtnis das Potenzial, wieder in die Erfahrungserinnerungen gegenwärtiger und zukünftiger Individuen, Gruppen und Generationen zurückzufließen und diese damit neu zu »inkarnieren « bei denen, die als Zeitzeug*innen an den Ereignissen teilgenommen hatten, aber auch bei denen, die zur Zeit der Ereignisse gerade erst oder noch gar nicht geboren waren. Assmann (ebd., 215) spricht in diesem Zusammenhang von der zweiten und dritten Generation, die in der konsequenten Genealogie derjenigen Ereignisse steht, die ihre Identität geprägt haben, aber außerhalb der Reichweite ihrer bewussten und selbstgewonnenen Erfahrung. So sind die Erinnerungen an die 1990er Jahre allgegenwärtig, als Teil eines Erfahrungs- und eines Generationengedächtnisses. Assmann $(2014,14)$ definiert diese Omnipräsenz als »Noch-Gegenwart der Vergangenheit«.

Darüber hinaus spielt, so ist hinzuzufügen, bei der DDR- und Vereinigungserfahrung eine »Zwischen- und Zwittergeneration « eine Rolle, nämlich ostdeutsche Jugendliche, die im Alter von 12 bis 15 Jahren die deutsche Wiedervereinigung erlebten, in ihrer frühen Sozialisation mit Ost-Identität ausgestattet wurden, die noch von antifaschistischen Helden, kommunistischen Ritualen und SED-Insignien geprägt waren (vgl. Wolfrum 2006, 495) und dann plötzlich die »ersten Wessis aus Ostdeutschland« wurden, wie es die Autorin Jana Hensel (2017 [2002], 166) formulierte.

In dieser Arbeit wird folglich unterschieden zwischen drei Generationen: der Generation der (damals erwachsenen, aktiven) heutigen Zeitzeug*innen, der »Wende- oder Zwischengeneration« und der beziehungsweise den »Nach-Wende-Generation(en)«.

\subsection{Entwicklung lokaler Fernsehsender in der DDR und in Sachsen}

Während die Grundstrukturen des staatlichen DDR-Fernsehens bis zum 31. Dezember 1991 bestanden, hatten die ab 1990 entstandenen lokalen Fernsehsender eine dreifache Genese: a) Ihre technischen und teilweise auch personellen Wurzeln lagen im letzten Jahrzehnt der DDR, in den sogenannten »Antennengemeinschaften $\ll ;{ }^{10} \mathrm{~b}$ ) sie gingen

10 Die Kabelnetze der Antennengemeinschaften verbanden in der Endphase der DDR hunderte, tausende und mitunter bis zu 50000 Haushalte in Wohnblöcken 
teilweise aus der Bürgerrechtsbewegung in der DDR und der unmittelbaren Phase nach dem Fall der Mauer hervor; c) diese beiden Wurzeln waren teilweise miteinander verflochten.

Einige Beispiele für diese Wurzeln sollen nun näher dargestellt werden: In den hügeligen Regionen Sachsens, insbesondere im Erzgebirge, in der Sächsischen Schweiz, im Vogtland und im Zittauer Gebirge, erlaubte die Topographie nicht einmal den Empfang eines oder gar beider DDR-Fernsehprogramme. So war es etwa seit Mitte der 1980er Jahre ein staatlich akzeptiertes Verfahren, eine Höhenlage für eine »Antennenkopfstation « technisch zu vermessen und zu verorten, von der aus die Bürger*innen in unbezahlter Arbeit selbst Kabelnetze errichteten. Die Deutsche Post lizenzierte diese Stationen. Als Beispiel sei die Antennengemeinde Chemnitz/Ebersdorf genannt, die bereits zu Weihnachten 1984 stolz war, West-TV zu empfangen (vgl. Antennengemeinschaft Chemnitz/Ebersdorf, Chronik o.J.). Neben der Möglichkeit, das DDR-Fernsehen auch in abgelegenen und in Bergregionen zu bekommen, war es ein willkommener Nebeneffekt, manchmal sogar der Hauptgrund, dass man öffentlich-rechtliche oder kommerzielle Fernsehprogramme aus Westdeutschland sehen konnte (vgl. Antenne Meuselwitz).

Viele noch heute bestehende örtliche Kabelanlagen stammen daher aus DDR-Zeiten. Um eine Genehmigung zu erhalten, musste lediglich nachgewiesen werden, dass das DDR-Fernsehen irgendwo in der Kommune nicht optimal zu empfangen war (vgl. Staud 1996). Ein weiterer Hintergrund der geduldeten Kabelsysteme bestand auch darin, dass der Empfang der West-Programme so besser kontrolliert werden und man gegebenenfalls ganze Dörfer relativ leicht abschalten konnte.

Eine wesentliche Rolle für die Entwicklung des Lokalfernsehens in Sachsen spielten vier internationale technische Entwicklungen zu Beginn der 1980er Jahre: erstens die Entwicklung von Personal Computern (Apple II ab 1977, IBM 5150 ab 1981) und Spielekonsolen (Commodore $64 \mathrm{ab}$ 1982), zweitens die magnetische Bild- und Tonaufzeichnung per "Camcorder« (ab 1983) auch in Händen von Amateur*innen, die das »Fernsehen von nebenan« ermöglichen und befruchten sollten, drittens die Verkabelung per Kupfer- und Glasfaserkabel (seit Mitte der 1980er

mit Fernseh- und Radioempfang. Dies waren nichtstaatliche Einheiten und ihre Mitglieder waren durch einen sozialen, gemeinschaftlichen und technischen Pioniergeist motiviert. 
Jahre) und viertens die Entwicklung des Satellitenfernsehens seit 1987 (TV-Sat 1) und 1989 (TV-Sat2 sowie DFS Kopernikus).

Auf der Grundlage dieser Technologien und der dispersen, lokalen Netz-Infrastruktur entwickelten sich in der DDR örtliche Antennengemeinschaftsanlagen, aus denen Lokal-TV-Stationen entstanden. Hier zwei Beispiele: Bereits 1986 sollen nach unserem jetzigen Erkenntnisstand in Marienberg-Goldkindstein im Erzgebirge die ersten selbstproduzierten lokalen Programme mit Stand- und Bewegtbildinhalten begonnen haben. 1979 wurde der erste Antrag gestellt, Anfang 1981 war Marienberg im Erzgebirge auf private Initiative einer Antennengemeinschaft verkabelt, 1984 wurde die Antennenanlage (Kabel-Kopfstation) auf dem Mühlberg aufgebaut, und am 3. August 1984, mehr als fünf Jahre vor dem Fall der Mauer, stellte Günter Rötzer beim Rat des Kreises Marienberg einen Antrag auf »Kabelrundfunk in der Region Marienberg«, der nie abgelehnt oder genehmigt wurde (vgl. Rötzer 1984). Er fand dabei tolerierende Unterstützung vom Rat des Kreises Marienberg und des VEB Federnwerk Marienberg. Seit 1986 wurde im Kabelnetz der Stadt Marienberg auf Sat.1 eine Art elektronische Zeitung oder Teletext mit lokalen Inhalten ausgestrahlt. ${ }^{11}$ Diese Inhalte erzeugte man auf einer umprogrammierten Spielkonsole (Laser 210-BK, seit 1984 auf dem Markt). ${ }^{12}$ Aber die Einrichtung eines eigenen Kabelkanals dauerte bis November 1989, wobei nur wenige Videoproduktionen im Rahmen von Videotextnachrichten, Veranstaltungsankündigungen und lokaler Werbung stattfanden. Bis 1991 wurde das Lokalfernsehen, das zum größten Teil ein als Schleife gesendetes Teletext-»Programm« und nur wenige Videos enthielt, in etwa 15 sächsischen Gemeinden verbreitet. Die Zulassung durch die Sächsische Landesmedienanstalt (SLM) erfolgte 1992; heute ist das Programm der Regionalfernsehen Mittelerzgebirge MEF $\mathrm{GmbH}$ nahezu im gesamten Mittleren Erzgebirgskreis zu empfangen.

Eine ähnlich interessante Entwicklung durchlief die Großantennengemeinschaft Burgstädt. Ab 1980 entwickelten sich im Stadtgebiet von Burgstädt, etwa 20 Kilometer von Chemnitz (damals Karl-Marx-Stadt) entfernt, und den angrenzenden Nachbargemeinden aus Bürgerinitiativen fünf Antennengemeinschaften, die sich 1984 unter einem Dach zusammenschlossen und Ende 1989 auf die Herausgabe der »Burgstädter Kabelzeitung« einigten. Diese »Kabelzeitung« erhielt eine Sendelizenz

11 Teletext beziehungsweise Videotext befand sich seit 1980 in der Bundesrepublik im Regelbetrieb von immer mehr ARD-Anstalten (vgl. Chronik der ARD 1978).

12 https://www.mef-line.de/über-uns/entwicklung.html 
von der DDR-Regierung unter Hans Modrow und wurde ab Februar 1990 ausgestrahlt. Ein Rechtsnachfolger, »RG Regional-TV«, existiert bis heute. ${ }^{13}$

Das Lokalfernsehen in Burgstädt und das Beispiel des heutigen »MittelErzgebirgsFernsehens « in Marienberg zeigen die Kontinuität von einer DDR-Kabelnetzgemeinschaft in einigen großen Wohnblöcken zu den frühesten Lokalfernsehsendern im vereinten Deutschland (vgl. Großantennengemeinschaft Burgstädt und Großgemeinschaftsantennenanlage Marienberg-Goldkindstein 2020). Die späten DDR-Jahre und die frühe Transformationszeit ermöglichten kleineren und größeren Bürgerinitiativen - wenn man die Antennengemeinschaften auch als solche bezeichnen will - unterhalb des staatlichen Radars Rundfunk zu veranstalten. Bis zur Etablierung einer neuen Zulassungsbehörde kann man diese Initiativen als »Piratensender« bezeichnen (vgl. auch Kretzschmar und Steinmetz 2020, 89).

Die Sächsische Landesanstalt für privaten Rundfunk und neue Medien (SLM) wurde Ende 1991 gegründet, nahm am 2. Januar 1992 in Dresden ihre Tätigkeit auf und vergab seit Spätsommer 1992 die ersten lokalen Rundfunklizenzen (vgl. Kühn 2003, 95-98), die erste am 27. August 1992 an das »Kabeljournal« in Röhrsdorf bei Chemnitz. Bis Ende 1995 hatte die SLM Lizenzen für etwa 68 lokale Fernsehsender vergeben, davon um die 40 für Kabelgemeinschaftsanlagen mit »Telezeitung $\ll \cdot{ }^{14} \mathrm{Als}$ Indikator für einen dann beginnenden Konsolidierungsprozess dieses kurzzeitigen Gründungsbooms liefen bis 1999 bereits wieder 21 Sendelizenzen aus oder wurden zurückgegeben.

\subsection{Programm-Dimensionen}

In Sachsen entstand in der ersten Hälfte der 1990er Jahre die reichhaltigste lokale Fernsehlandschaft in ganz Deutschland. Diese Programme bestanden in weiten Teilen aus lokalen und subregionalen Teletext-Informationen, von ihnen auch als »Fernseh-Zeitung « bezeichnet, und lokaler Videotext-Werbung. Ein geringer Teil des meist in Endlosschleifen laufenden 24-Stunden-Angebots bestand aus klassischen bewegten Fernsehbildern, manchmal mit werktäglich aktualisierten Magazinen,

13 https://gag-burgstaedt.de/history.html

14 Eigene Berechnungen, basierend auf N.N. (Richter). Veranstalterübersicht Fernsehen - Erstzulassung bis 1995. SLM, interne Quelle, undatiert. 
meist aber mit nur einmal wöchentlich aktualisierten Beiträgen. In den ersten Jahren gehörten auch PR-Videos westdeutscher Unternehmen, internationale Musikvideos, Animationsfilme und Werbung für lokale Geschäfte und Händler zum Bewegtbild-Programm.

Eine genaue Berechnung der Programmdimensionen in Stunden oder Kassetten in der ersten Hälfte der 1990er Jahre in Sachsen ist meist mangels einer archivarischen Erfassung schwierig. In den Jahren 1990, 1991 und drei Viertel des Jahres 1992, also in der Zeit vor der Lizenzierung, gab es weniger als zehn lokale Stationen, bis Ende 1995 etwa 40. Eigene vorsichtige Berechnungen gehen von bis zu 1000 Programmstunden Bewegtbild von 1990 bis 1995 aus.

In Sachsen wurden zwischen 1990 und 1999 zahlreiche lokale Fernsehsender gegründet und auf einer zeitlich und räumlich sehr unterschiedlichen Basis betrieben. Zudem wandte man professionelljournalistische und westliche kommerzielle Programmformate sehr unterschiedlich an. Alle Programme waren - mehr oder weniger Learning-by-doing-Projekte, sowohl aus journalistischer als auch aus technischer und fernseh-ästhetischer Sicht. Gerade die dahingehende »Unbedarftheit« vieler ostdeutscher Macher*innen und ihrer überlieferten Programme trägt heute ganz wesentlich zur Authentizität und zum historischen Quellenwert des Materials bei. Manchmal sind hier Direct-Cinema-Berichte im besten Sinne zu finden, die kaum eine Differenz zwischen den »Macher*innen« als Nachbar*innen und den Dargestellten erkennen lassen. In den Kleinstädten und ländlichen Gemeinden ist ein sehr freier Umgang mit der Programmzeit festzustellen. Im Hinblick auf den kulturellen Wert der Programme und ihren Beitrag zum authentischen Bild des Alltags und seiner Veränderungen bildet all dies ein wesentliches Unterscheidungs- und Qualitätsmerkmal gegenüber den Programmen des öffentlich-rechtlichen Fernsehens.

\subsection{Auswahl sächsischer Lokalfernsehsender}

Im Folgenden werden Struktur und Geschichte der Sender kurz erläutert, deren Programme genauer untersucht wurden und die mit Programmausschnitten Eingang in die im Projekt realisierten Wiederaufführungen von Lokal-TV-Beiträgen gefunden haben. 
Tab. 1: Ausgewählte sächsische Lokalfernsehsender (Zusammenstellung auf Basis des gegenwärtigen Kenntnisstandes der Autor*innen).

\begin{tabular}{|c|c|c|}
\hline Name & Sendezeitraum & Besonderheiten \\
\hline $\begin{array}{l}\text { Stadtfernsehen } \\
\text { Leipzig }\end{array}$ & $\begin{array}{l}\text { März } 1990 \text { bis } \\
1993\end{array}$ & $\begin{array}{l}\text { Ältestes Lokalfernsehen in Leipzig. Das Nachrichten- } \\
\text { magazin »Studio Leipzig « wurde in einem gläsernen } \\
\text { Studio mitten in der Stadt präsentiert, als Zeichen für } \\
\text { Medientransparenz - in ausdrücklichem Gegensatz zu den } \\
\text { DDR-Medien. }\end{array}$ \\
\hline $\begin{array}{l}\text { Regionalfern- } \\
\text { sehen Mittel- } \\
\text { erzgebirge MEF } \\
\text { GmbH }\end{array}$ & 1986 bis heute & Siehe Abschnitt 2.4. \\
\hline $\begin{array}{l}\text { Hoy-TV, später } \\
\text { Elsterwelle } \\
\text { TV (2000 bis } \\
\text { 2018), heute } \\
\text { Lausitzwelle TV }\end{array}$ & $\begin{array}{l}\text { Januar } 1993 \text { bis } \\
\text { heute }\end{array}$ & $\begin{array}{l}\text { Sendete zunächst für die ostsächsische Stadt Hoyers- } \\
\text { werda, dann auch für die Stadt Kamenz. Tägliche, viertel- } \\
\text { stündige Nachrichten folgten ab September 1993. Dazu } \\
\text { kamen drei Magazine am Montag, Mittwoch und Freitag } \\
\text { mit Themenschwerpunkten. Betreibergesellschaft war und } \\
\text { ist die SGS Rundfunkgesellschaft GmbH. }\end{array}$ \\
\hline LTV & 1992 bis 1994 & $\begin{array}{l}\text { Gegründet von Peter Vonstein, in der DDR Leiter des } \\
\text { Leipziger Bezirkskabinetts für Kulturarbeit und Anfang der } \\
\text { 1990er Jahre Produzent (»Stein-TV «) der Hallervorden- } \\
\text { Show auf Sat.1. Vonstein gründete die Produktionsfirma } \\
\text { Neues aus Sachsen GbR als Muttergesellschaft für das } \\
\text { dritte Leipziger Lokalfernseh-Programm LTV. Dieses bekam } \\
\text { ein Fenster auf der Frequenz von Vox zwischen } 17 \text { und } 20 \\
\text { oder } 21 \text { Uhr. Programmschwerpunkte waren Kultur (bei- } \\
\text { spielsweise Kirchenporträts), Live-Diskussionen zu Politik- } \\
\text { und Kulturthemen, Sport und Nachrichten. }\end{array}$ \\
\hline $\begin{array}{l}\text { E-R-tv / } \\
\text { Erzgebirge } \\
\text { Regional TV, } \\
\text { Marienberger } \\
\text { Fernsehen }\end{array}$ & $\begin{array}{l}\text { Beginn unbe- } \\
\text { kannt, SLM-Lizenz } \\
\text { von März } 1994 \text { bis } \\
\text { März } 2007\end{array}$ & $\begin{array}{l}\text { Der Sender produzierte alle } 14 \text { Tage ein dreistündiges, } \\
\text { nicht formatiertes Programm, das in Endlosschleife in } \\
\text { Kabelanlagen in Ansprung, Lengefeld, Zschopau und } \\
\text { Zöblitz lief. } 1995 \text { ließ der Zschopauer Bürgermeister das } \\
\text { Programm wegen »Unstimmigkeiten « mit dem Programm- } \\
\text { chef abschalten und verwies Letzteren mit seiner Kamera } \\
\text { aus dem Stadtrat. Die SLM musste gegen die Abschaltung } \\
\text { des Programms einschreiten. }\end{array}$ \\
\hline
\end{tabular}




\begin{tabular}{|c|c|c|}
\hline Name & Sendezeitraum & Besonderheiten \\
\hline $\begin{array}{l}\text { Laubuscher } \\
\text { Heimatkanal }\end{array}$ & $\begin{array}{l}\text { März } 1992 \text { bis } \\
2009\end{array}$ & $\begin{array}{l}\text { Betreiber war der evangelische Pfarrer Gerd Simmank. Er } \\
\text { realisierte ein Fernsehprogramm, das Ereignisse in der } \\
\text { kleinen Lausitzer Gemeinde Laubusch (gehört heute zur } \\
\text { Stadt Lauta) ungeschnitten und unformatiert abbildete, } \\
\text { um Öffentlichkeit herzustellen und Bürger*innenbetei- } \\
\text { ligung zu ermöglichen. Zusätzlich strahlte er rund } 3600 \\
\text { Morgenandachten aus. Pfarrer Simmank finanzierte alles } \\
\text { aus eigener Tasche (mehr in Kretzschmar und Steinmetz } \\
\text { 2020, 93). }\end{array}$ \\
\hline Kanal X & $\begin{array}{l}\text { März } 1990 \text { bis } \\
\text { Anfang } 1991\end{array}$ & $\begin{array}{l}\text { Gegründet in Leipzig von den westdeutschen Videokünst- } \\
\text { lern Ingo Günther und Norbert Meissner sowie den ost- } \\
\text { deutschen Anja-Christin Remmert (Autorin) und Jörg Seyde } \\
\text { (Kunsthistoriker). Eingetragen als gemeinnütziger Verein. } \\
\text { Beim Runden Tisch Leipzig beantragte der Verein in der } \\
\text { Endphase der DDR eine Sendelizenz, die ihm der Medien- } \\
\text { kontrollrat aber nicht erteilte. }\end{array}$ \\
\hline $\begin{array}{l}\text { RFV / VRF } \\
\text { Vogtland } \\
\text { Regional } \\
\text { Fernsehen }\end{array}$ & $\begin{array}{l}\text { Januar } 1994 \text { bis } \\
\text { Dezember } 2015\end{array}$ & $\begin{array}{l}\text { Sendete in Kabelanlagen in Plauen, Auerbach, Klingenthal, } \\
\text { Markneukirchen, Reichenbach und dem Oberen Vogtland } \\
\text { wöchentlich drei Stunden aktuelles Programm mit Schwer- } \\
\text { punkten auf Sport, Wirtschaft und Regionalkultur. Seine } \\
\text { Kulturprogramme, unter anderem die Rubrik »Geschichte } \\
\text { aus Stein«, waren ebenso beliebt wie »Der Blumenstrauß } \\
\text { der Woche « für »verdiente VogtländerInnen « und »Vogt- } \\
\text { länder filmen selbst«. Das Programm wurde für seine } \\
\text { Qualität mit vielen Preisen ausgezeichnet. }\end{array}$ \\
\hline Torgau-TV & $\begin{array}{l}\text { April } 1995 \text { bis } \\
\text { heute }\end{array}$ & $\begin{array}{l}\text { Verbreitet in den Kabelnetzen Torgau, Beilrode, Arzberg } \\
\text { und Zinna. Das Programm bestand aus einer wöchent- } \\
\text { lichen einstündigen Sendung mit einem langen Beitrag, } \\
\text { einer »Wochenschau « und einem »Polizeireport«. Es } \\
\text { enthielt auch Reportagen, Dokumentationen, Interviews, } \\
\text { Kommentare, Ratgebersendungen und Veranstaltungs- } \\
\text { tipps. }\end{array}$ \\
\hline $\begin{array}{l}\text { eff3 Frei- } \\
\text { berg (Erstes } \\
\text { Freiberger } \\
\text { Fernsehen - } \\
\text { 3. Kanal) }\end{array}$ & $\begin{array}{l}\text { Juni } 1994 \text { bis Juni } \\
2015\end{array}$ & $\begin{array}{l}\text { Kabel-Fernsehprogramm für Freiberg, Conradsdorf, Naun- } \\
\text { dorf, Hilbersdorf, Halsbrücke, Krummenhennersdorf, Klein- } \\
\text { waltersdorf, Zug, Weißenborn und Großschirma. Täglich } \\
\text { zu jeder ungeraden Stunde konnten die angeschlossenen } \\
\text { Haushalte das 40-minütige, wöchentlich aktualisierte } \\
\text { Programm empfangen. Wie bei anderen lokalen Fernseh- } \\
\text { programmen wechselten sich in der Programmschleife } \\
\text { Fernseh- und Videotext-Teile ab. }\end{array}$ \\
\hline
\end{tabular}




\begin{tabular}{|c|c|c|}
\hline Name & Sendezeitraum & Besonderheiten \\
\hline $\begin{array}{l}\text { Euro-Regional- } \\
\text { TV / eRtv } \\
\text { Görlitz }\end{array}$ & 1992 bis 2017 & $\begin{array}{l}\text { Programm für die Landkreise Görlitz, Bautzen und } \\
\text { Zgorzelec (Polen), das von Beginn an grenzüberschreitend } \\
\text { konzipiert war. Die technische Reichweite lag bei bis zu } \\
300000 \text { Zuschauer*innen, davon etwa } 80000 \text { in Polen. } \\
1992 \text { Beginn als »Bildschirmzeitung « (Videotext). Später } \\
\text { kamen das deutsch-polnische Magazin »Nachbarn« und } \\
\text { das Wirtschaftsregionenmagazin »Zukunft Heimat« dazu. }\end{array}$ \\
\hline Eilenburg-TV & $\begin{array}{l}\text { Februar } 1995 \text { bis } \\
\text { Februar } 2016\end{array}$ & $\begin{array}{l}\text { Wurde von der im Oktober } 1994 \text { von Karl-Heinz Hempel } \\
\text { und Andrea Heyn gegründeten Media Concept GmbH für } \\
\text { zunächst } 6000 \text { Kabelhaushalte in der nordsächsischen } \\
\text { Kleinstadt (knapp } 20000 \text { Einwohner*innen) veranstaltet; } \\
\text { Sendelizenz der SLM am 8. Februar 1995. Sendestart im } \\
\text { Juli } 1995 \text { zunächst mit Videotext-Schleifen, dann ab dem } \\
\text { Mulde-Hochwasser im September } 1995 \text { auch mit Video- } \\
\text { berichterstattung. Hempels Erfahrungen resultierten aus } \\
\text { dem alteingesessenen Fernsehgeschäft seines Vaters und } \\
\text { Heyn war eine komplette Seiteneinsteigerin als Diplom- } \\
\text { ingenieurin für Betontechnologie. }\end{array}$ \\
\hline
\end{tabular}

\section{$3 \quad$ Methoden der Untersuchung}

Entsprechend den in der Einleitung aufgeworfenen Forschungsfragen, 1) die Relevanz für eine heutige Reaktivierung direkter sowie indirekter, mediatisierter Erfahrungen zu untersuchen und 2) die Wurzeln heutiger aus der Transformation resultierender Problemthemen aufzuspüren und die bisherige »Nachwende«-Geschichtsschreibung kritisch zu hinterfragen, wurde für dieses Projekt ein Methodenmix entwickelt. Darin bekommen heutige Zuschauer*innen (drei Generationen) Programme aus der Sattelzeit kontextualisiert vorgeführt. Dann werden die heutigen Nutzer*innen zur (Re-)Aktivierung ihrer Erinnerungen und inzwischen gemachten Erfahrungen befragt. Aus historischer, politischer, soziologischer, medienwissenschaftlicher und Oral-HistoryFachliteratur wurden zuvor thematische Kategorien entwickelt, die der teilweise quantifizierenden, überwiegend aber qualitativen Analyse von Programmen und Reaktionen auf die Programme zugrunde liegen.

Es handelt sich wegen seines noch nicht gänzlich überschaubaren Umfangs um ein Projekt in progress. Es befindet sich auf dem Weg zwischen einerseits deduktiv abgeleiteten Kategorien und Subkategorien wie auch andererseits induktiv aus dem Gesprächs- oder Interviewma- 
terial gewonnenen Ergebniskategorien und Themenclustern. Hinzu kommt, dass die beiden Autor*innen implizite Hypothesen haben - bedingt durch ihre Ost- beziehungsweise Westsozialisation, Innen- oder Außensicht und -Erfahrung sowie ihre Geschlechts- und Generationenunterschiede -, die erst im Laufe des Forschungsprozesses explizit werden. Das Projekt ist in der gegenwärtigen Phase in seiner Abfolge und seinem Aufbau weiterhin auf Offenheit und Hypothesengewinnung angelegt.

Folgende Methoden wurden angewandt:

- Deduktion von zwölf Themenkategorien für die Analyse der Programme aus der Literatur. ${ }^{15}$

- Quantitative und qualitative Analyse lokaler sächsischer Fernsehprogramme der Jahre 1990 bis 1995.

15 1. System-Transformation übergreifend: Überführung sozialistischer Strukturen in kapitalistische; 2. Politische Transformation: Aufbau neuer politischer, demokratischer und rechtsstaatlicher Strukturen; 3. Ökonomische Transformation: Währungsaufwertung und Einkommensangleichung - dann aber der Kollaps der Wirtschaft und die Bedürftigkeit nach Alimentation, Anpassung der Realitäten unter dem Druck erhöhter Ansprüche und verschlechterter Bedingungen; 4. Kulturelles Leben: Schließung und Neu-Etablierung von Bildungs-, Kultur- und Sozialeinrichtungen; 5. Arbeitsleben: Schließung (»Abwicklung«) von Industrie-, Landwirtschafts- und Handelsbetrieben, Arbeitsplatzverlust, Neu-Orientierung auf Arbeitsstrukturen und -profile, Konkurrenz, Leistungsanforderungen, erste Streiks, Arbeitsemigration nach Westdeutschland; 6. Soziale und allgemeinmenschliche Lage: Verlust erworbener sozialer und ethischer Standards und Ziele, Neuorientierung, Rollen von Familie, Freundeskreis, Nachbarschaft und Arbeitskolleg*innen, Übernahme von mehr Eigenverantwortung, Generationen-Sichtweisen; 7. Umgang mit der (eigenen) Vergangenheit: Entdecken, Aufdecken, Diskutieren; altes und neues (politisches) Personal, Schuldfragen; 8. Heimat: Traditionen, Brauchtum, Feste; regionale/lokale Dialekte und Speisen; 9. (Verkehrs-)Infrastruktur, (Landschafts-)Architektur: Verkehrsentwicklung, Stadtplanung und Restaurierung, Ökologie und Umweltschutz, Wandel der Dörfer, Städte und Landschaften, Freizeit und Tourismus; 10. Das Eigene und das Fremde: neue Reisemöglichkeiten, Entdecken »fremder« Länder und Kulturen, neue Erfahrungen und alte Vorurteile; neue ausländische Restaurants, Speisen und Getränke; Ausländerfeindlichkeit, »Besser-Wessis« - »JammerOssis«; 11. Phasen der Erfahrung mit der Vereinigung: Euphorie, Ernüchterung und Desillusionierung, falsche und enttäuschte Hoffnungen, Integration und Pragmatik; 12. Neue Medien-Strukturen und -Erfahrungen: West-Dominanz im Mediensektor, Nachahmung westdeutscher Programmformate und Medienpraktiken, neue Medienmöglichkeiten und -freiheiten. 
- Kontextualisierende Präsentationen ausgewählter Programme an mehr als zwölf Standorten in Sachsen mit anschließenden Fokusgruppen-Gesprächen.

- Qualitative Analyse der Fokusgruppen-Gespräche entsprechend den thematischen Kategorien und Unterkategorien.

- Macher*inneninterviews.

Bisher (Stand: März 2021) wurden etwa 250 Programmstunden von zwölf Lokalfernsehsendern untersucht und Fokusgruppen-Gespräche mit etwa 200 Teilnehmer*innen sowie zehn Macher*inneninterviews geführt.

\section{Einige Zwischenergebnisse}

Aus den zwölf Untersuchungskategorien werden im Folgenden zwei ausgewählt, die sich besonders klar aus der Auswertung der bisherigen Fokusgruppen-Gespräche an den über Sachsen verteilten Veranstaltungsorten ergaben: erstens die Umbrüche im Arbeitsleben und deren erlebte Auswirkungen und zweitens das Eigene und das Fremde (OstWest). Diese Fokusgruppen-Gespräche wurden leitfadengestützt durch zwei Moderator*innen jeweils im Anschluss an einen einleitenden Vortrag und die Präsentation einer teilweise regionalspezifischen Auswahl von Programmclips geführt und anonymisiert aufgezeichnet. ${ }^{16}$ Bei der Auswertung der Fokusgruppen-Gespräche wurde auf die interpretativreduktive Methode von Lamnek (1995; 1998; vgl. Lamnek und Krell 2016) zurückgegriffen.

\subsection{Umbrüche im Arbeitsleben und deren erlebte Auswirkungen (als Ängste)}

Durch die Fernsehclips wurden bei den Teilnehmer*innen sowohl damalige, aber auch gegenwärtige Zukunfts- und Existenzängste, Traumatisierungen sowie die Gefühle der Selbst- und Fremdscham re-aktualisiert.

16 Die Transkriptionen wurden auf Basis digitaler Tonmitschnitte und Videoaufzeichnungen zur Zuordnung eventuell wiederkehrender Sprecher*innen angefertigt. Der mündliche Sprachduktus wurde beibehalten, aber behutsam in eine lesbare Fassung redigiert. 
Diese wurden generell ausgelöst durch den abrupten Bruch mit den eingespielten Gewohnheiten des Alltags, speziell aber vor allem durch gravierende Einschnitte im Arbeitsleben nahezu eines und einer jeden. Ein erheblicher Teil dieser Gefühle ist heute noch virulent, unabhängig davon, ob die Zeitzeug*innen eher eine positive oder negative Bilanz der Vereinigung für sich ziehen. Es wurde deutlich, dass die genaue lokale Provenienz der Clips zwar eine gewisse Rolle spielte; aber es zeigte sich vor allem, dass die primären, »inkarnierten « Erfahrungen der Zeitgenoss*innen, an welchem Ort auch immer gewonnen, durch die mediatisierten, »exkarnierten« Erfahrungen mit den ausgewählten Clips aus anderen Orten Sachsens revoziert, aktualisiert und (quasi auf einem zweiten methodischen Weg) bestätigt, beglaubigt und verfestigt wurden. Das ließe sich entweder damit begründen, dass die Forscher*innen eine »typische«, repräsentative Programmauswahl getroffen hatten oder dass diese inkarnierten Erfahrungen eben überall in Sachsen, wenn nicht darüber hinaus in ganz Ostdeutschland, gemacht wurden. Wir vermuten Letzteres.

Hier einige Zitate aus den Gesprächen, die sich auf die zuvor gesehenen Beispiele beziehen:

Die Angst, die Angst, die dann gerade in den ersten Jahren nach der Wende war: »Was wird denn jetzt? « Also ich habe mich gefragt, und viele andere, »wie geht es denn weiter? « Die Westmark war erst einmal schön und dass man in den Urlaub, vielleicht, fahren konnte. Aber, was wird? Es hatte sich dann angedeutet, erst die Betriebe haben so eine Übergangsgesellschaft gemacht, dann wurden Lehrgänge angeboten, dann ABM. ${ }^{17}$

Ich habe schon zu DDR-Zeiten mit geistig und mehrfach behinderten Kindern gearbeitet und bin jetzt 41 Jahre an der gleichen Stelle. [...] Wir haben eigentlich insofern, naja, den Umbruch relativ still erlebt. Nur, dass die Ängste immer sehr geschürt waren, und die Ängste waren ähnlich wie in dem Film. [...] Behalten wir unseren Arbeitsplatz? [...] Wir [haben] ganz oft zusammengesessen, wenn irgendwelche Anbieter kamen, die sagten: »Wir übernehmen in einem halben Jahr die Schule, und dann seid ihr alle entlassen. «Also solche Phrasen haben wir durch. Es hat sich eigentlich immer wieder gezeigt, dass am Ende das gar keiner so umsetzen konnte wie geplant. [...] Das war immer ganz beängstigend, doch am Ende sieht man, dass die wirkliche Arbeit dann eben gezählt hat. Aber man hat im Grunde genommen die Ängste damals gesehen, und heute sind sie eigentlich immer noch da, nur anders, aber immer noch präsent. Und von Zeit zu Zeit kommt

17 Sprecher im Mehrgenerationenhaus Zwönitz (Erzgebirge) am 6. März 2020. $\mathrm{ABM}=$ Arbeitsbeschaffungsmaßnahme. 


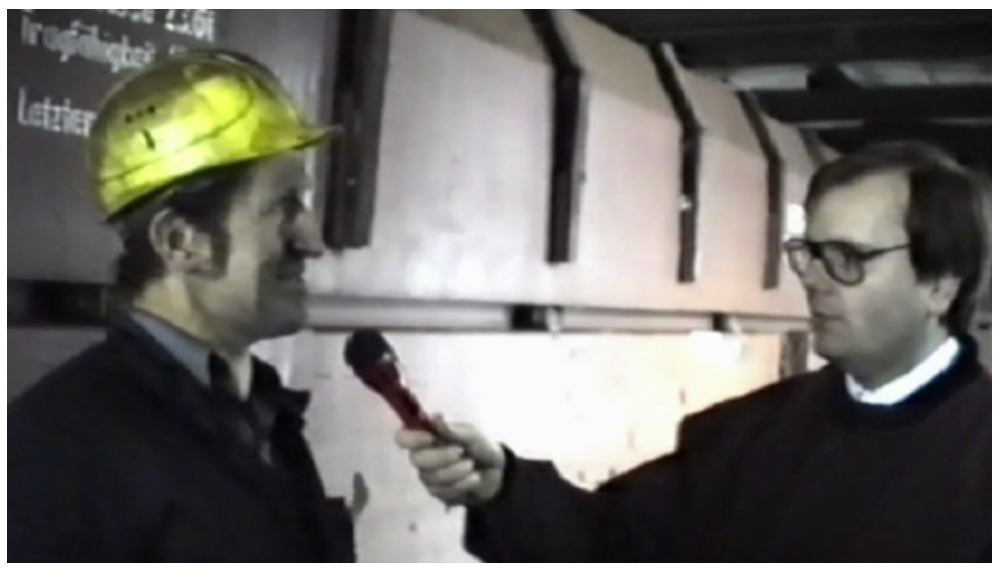

Abb. 3: Etwa 1000 Arbeitsplätze fallen weg, als Ende 1993 die Brikettfabrik in Laubusch (Lausitz) geschlossen wird. Unter den Arbeiter*innen herrscht Zukunftsangst. (C) Laubuscher Heimatkanal, 19. November 1993.

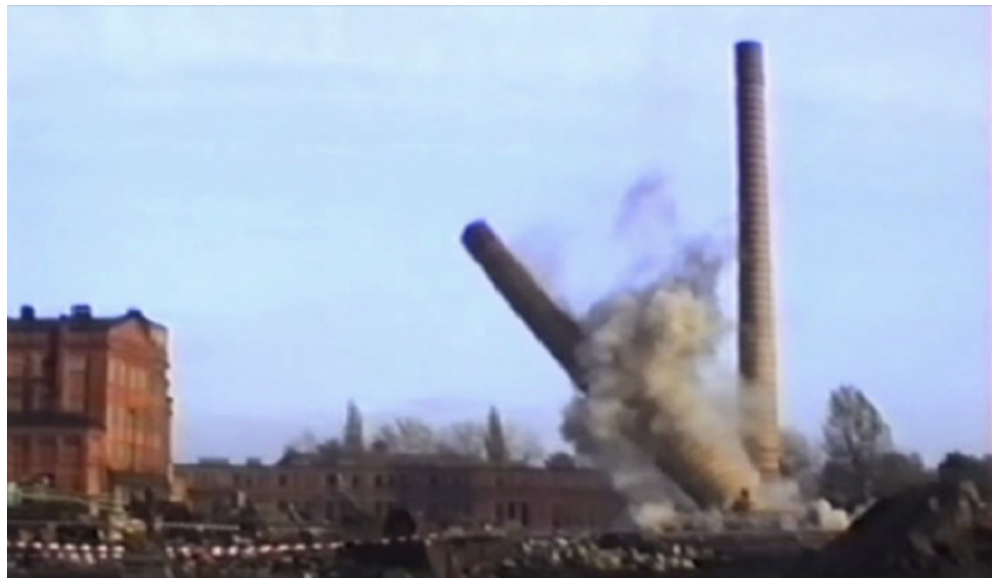

Abb. 4: Ikonisches Bild des Abbruchs: Sprengung auf dem Gelände der Brikettfabrik. (C) Laubuscher Heimatkanal, 19. November 1993.

dann irgend so ein Schatten, der irgendwas möchte, und den müssen wir dann immer wieder neu bekämpfen. ${ }^{18}$

Ich schätze das natürlich, jetzt die Welt bereisen zu können, was früher mal nicht möglich war. Aber ich habe halt so in Erinnerung, dass doch auch sehr

18 Sprecherin in der Stadtbibliothek Chemnitz am 11. Oktober 2019. 


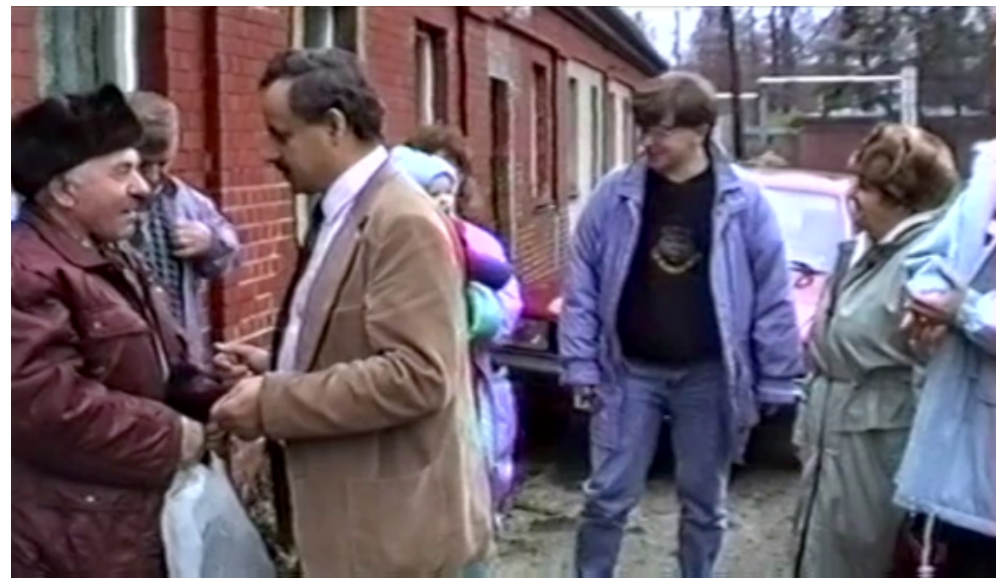

Abb. 5: Sozialer Sprengstoff im Laubuscher Lokalfernsehen: Dem kleinen Ort (etwa 5000 Einwohner) werden 1992 deutschstämmige Aussiedlerfamilien aus Kasachstan und der Ukraine zugewiesen, während kurz danach die Brikettfabrik geschlossen wird und knapp 1000 Menschen ihre Arbeit verlieren. (C) Laubuscher Heimatkanal, 6. November 1992.

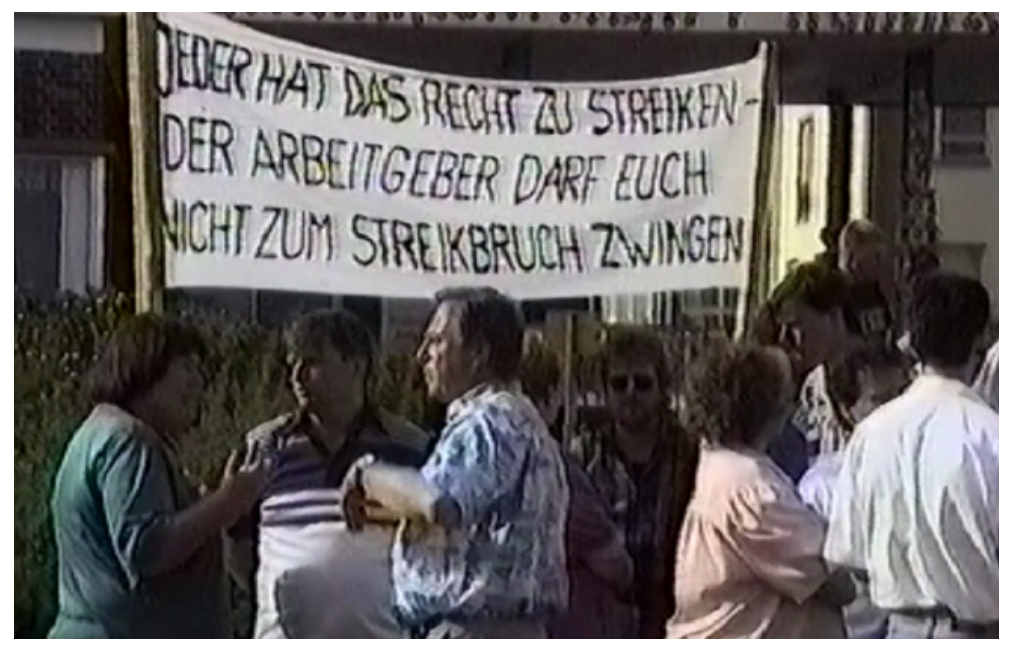

Abb. 6: Nach der Euphorie kam die Ernüchterung: Erste Streiks für Tarifverträge in Sachsen fanden im Frühjahr 1993 statt, hier am 4. Mai 1993 beim Druckmaschinenwerk MAN-Plamag in Plauen. Arbeiter*innen bekamen nur die Hälfte des Lohns bayerischer Metallfacharbeiter"innen. (C RFV/VRF Vogtland Regionalfernsehen, 4. Mai 1993. 


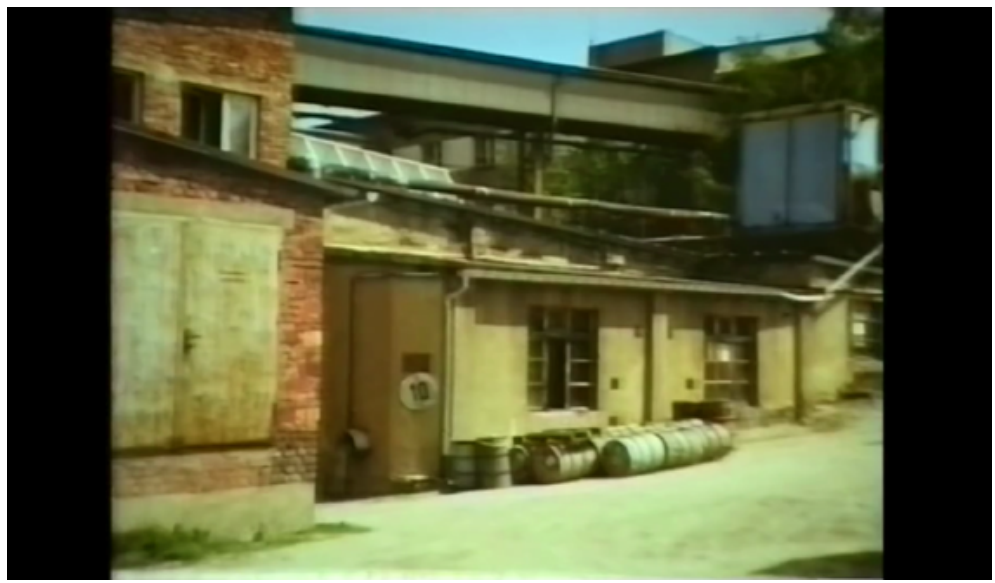

Abb. 7: Abbruch, Umbruch und Aufbruch in Auerbach (Vogtland): Aus dem »Suppina «-Werk wird »Knorr«-Suppen. (C) RFV / VRF Vogtland Regionalfernsehen, Februar 1993.

viel Verlustängste [...] und Existenzängste bestanden. Also, das ist das, was ich [...] aus dieser Zeit mitgenommen habe. ${ }^{19}$

Die folgenden Äußerungen beziehen sich auf Programmausschnitte, die den damals - nach Übernahme durch die großen westdeutschen Energiekonzerne - hoffnungsvollen Umgestaltungsprozess des Lausitzer Braunkohlereviers durch umweltfreundlichere Technik in Beziehung setzen zu der heutigen grundlegenden Umwandlung der Lausitz weg von der Braunkohle hin zu einer Hightechregion:

Da könnte einer ein Déjà-vu erleben. Wenn der mit 18 als Lehrling bei der LAUBAG dabei war und jetzt in »Schwarze Pumpe« arbeitet, sagt der: »Werde ich jetzt wieder arbeitslos, wie damals? $«^{20}$

[Dazu:] Es haben hier [in Hoyerswerda] über 70000 Menschen gewohnt, und jetzt sind es, glaube ich, in der Kernstadt nur noch 30000 . Das ist eben schon ein Schrumpfungsprozess, der geht parallel mit der wirtschaftlichen

19 Sprecher im Mehrgenerationenhaus Zwönitz (Erzgebirge) am 6. März 2020.

20 Sprecher im »studio eins« Hoyerswerda am 2. Juli 2020. Äußerung zum Thema "Schwarze Pumpe « und Braunkohletagebau damals sowie den Auswirkungen des Kohleausstiegsgesetzes vom Januar 2020 und der Digitalisierungsförderung in der Lausitz. Auf dem Areal des 1955 errichteten VEB Gaskombinats "Schwarze Pumpe« arbeitet heute ein 1992 errichtetes Braunkohle-Großkraftwerk. 
Entwicklung der Region einher. Falls es jetzt nicht gelingt, irgendeinen Ersatz zu schaffen, dann wird es noch wirklich schlimm kommen. Und insofern kann man schon die Ängste der Menschen verstehen. Also das ist bei uns natürlich auch, egal ob in der Zeitung oder halt auch Thema: Was wird passieren ${ }^{21}$

Die hier ausgewählten Beispiele belegen zwei durchgehende Trends in den Fokusgruppen-Gesprächen: zum einen, dass die in den Erinnerungen der Angehörigen der Zeitzeugen-Generation tief verwurzelten Erinnerungen und Gefühle durch die Fernsehclips reaktiviert wurden; und zum anderen, dass diese scheinbar verschütteten Kontexte auf die eigene Gegenwart bezogen und durchaus auch von einer Region auf die andere übertragen, also verallgemeinert werden.

\subsection{Das Eigene und das Fremde: Ost- und Westdeutsche}

Die zweite untersuchte Kategorie »Das Eigene und das Fremde«nimmt die scheinbaren Gewissheiten und Argumentationsmuster (im Sinne verfestigter Topoi) der letzten Jahrzehnte auf, nach denen sich die Welt des vereinten Deutschlands in »Besser-Wessis« und »Jammer-Ossis« einteilen ließe. Auch hier ging es darum, inkarnierte Erfahrungen anhand von historischen audiovisuellen Quellen überprüfen, bestätigen oder widerlegen zu lassen und damit als exkarnierte Erfahrungen entweder zu beglaubigen oder zu falsifizieren.

Hier wieder Beispiele aus den Fokusgruppen-Gesprächen, die sich auf die gerade zuvor gesehenen Fernsehclips beziehen und durchaus unterschiedliche reaktivierte Erfahrungen und heutige Positionen verdeutlichen:

Der einzige Gedanke, der mir jetzt beim Anschauen dieser Videoclips gekommen ist: dass einige Gedanken, die wir damals schon hatten, sich bestätigen im Rückblick. Gerade diese Konfrontation. Ich nenn' es jetzt mal »Konfrontation«, vielleicht ist es nicht immer eine Konfrontation im wahrsten Sinne des Wortes gewesen, aber es sind doch Welten aufeinandergeprallt, die sich im persönlichen Bereich zusammenfinden mussten oder auch nicht zusammengefunden haben, wenn wir Auseinandersetzungen mit Investoren [...] hatten. Ich denke manchmal, manche Sachen, die man uns Ossis als Vorurteile vorgeworfen hat, die bewahrheiten sich im Nachhinein eigentlich schon. In vielen Bereichen muss man im Nachhinein sagen:

21 Sprecher im »studio eins« Hoyerswerda am 2. Juli 2020. 
Abb. 8: Verteilung von Schokoriegeln auf dem Leipziger Marktplatz, Mitte März 1990. Der Beitrag heißt »Test the West.« (C) Stadtfernsehen / Studio Leipzig, März 1990.

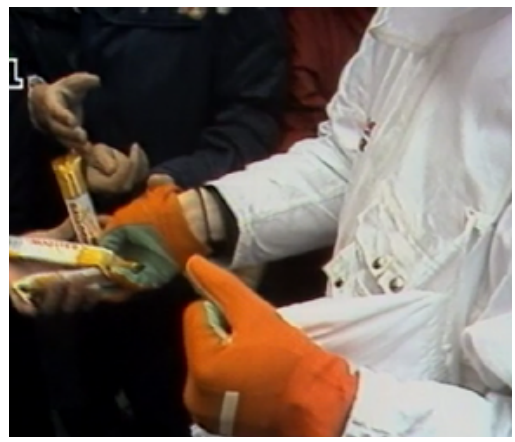

Ja, wir hatten vielleicht doch bewusst richtige Vorurteile beziehungsweise waren skeptisch. ${ }^{22}$

Das [die gezeigten Clips] spricht die Gefühle an. Ich bin eine Görlitzerin und auch schon etwas älter, ich hab' das alles miterlebt. Das ist unglaublich, was das bei mir ausgelöst hat. [...] Ich musste auch viel lachen hier, ich hab' auch geweint; das ist so, das betrifft einen ja selber. [...] Was ich erlebt hab' [...]: Wie die einzelnen kleinen Leute vor Ort im Westen hilfsbereit waren, wie die uns auch unterstützt haben, gegenseitig auch respektiert haben. Ich denke immer, die Kleinen, die miteinander zu tun hatten, die ohne wirtschaftliche Interessen irgendwas taten, damit konnte man ganz viel anfangen. $[\ldots]^{23}$

Wie man damals auch umgegangen ist miteinander: Ich hab' das selbst erlebt auf dem Marienplatz [in Görlitz], wie dort dieser riesige LKW von Milka stand und kostenlos diese Schokoladentafeln verteilt haben an unsre Görlitzer Bürger. Und wenn da eine runterfiel, die Leute sind auf allen v-ie-r-e-n gekrochen und haben das aufgehoben. Da hab' ich mir gedacht, was ist denn mit den Leuten los? Was geht hier vor sich? Wieso erniedrigen wir uns so? Wieso lassen wir uns so vereinnahmen? ${ }^{24}$ (Vgl. Abb. 8).

Mich hat es peinlich berührt, muss ich sagen, mit dem Wissen von heute natürlich. Wie wir da so nach so Zeitungen, so einem Mist da angestanden haben und uns die Bananen um die Ohren hauen haben lassen, furchtbar. Muss ich jetzt echt so sagen, das ist schlimm eigentlich. Aber gut, das war damals die Zeit. ${ }^{25}$ (Vgl. Abb. 9)

Wir haben dem Westen viel zu verdanken. Was wäre denn die Alternative gewesen, wenn der Westen gesagt hätte, wir nehmen euch nicht? Unser

22 Sprecherin in der Stadtbibliothek Chemnitz am 11. Oktober 2019.

23 Sprecherin in der Stadtbibliothek Görlitz am 10. Oktober 2019.

24 Sprecherin in Stadtbibliothek Görlitz am 10. Oktober 2019.

25 Sprecher im »studio eins« Hoyerswerda am 2. Juli 2020. 
Abb. 9: Verkaufvon West-Zeitungen auf dem Leipziger Marktplatz im März 1990. Im Beitrag findet vor allem die Bild-Zeitung reißenden Absatz. (C) Stadtfernsehen/Studio Leipzig, 16. März 1990.

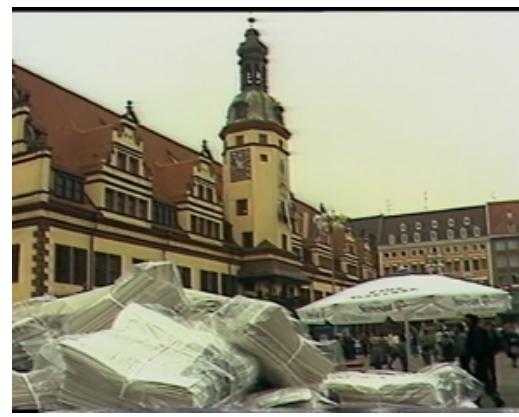

Geld war in der Welt wenig wert. Wir hatten wenig Rohstoffe; das Uran hat der Russe abgegriffen [...], weil das Uran eben ganz wertvoll war. Ich bin ein Kind des Erzgebirges und ich bin heute noch sehr dankbar, dass meine Kinder ihre Meinung sagen dürfen, dass sie, ohne Parteibuch und ihre Meinung nach dem Wind zu richten, aufs Gymnasium durften. ${ }^{26}$

Ich musste wie viele arbeitslos Gewordene nach der Wende aus dem Beruf ganz raus, vollkommen was Neues gemacht: Umschulung zum Altenpfleger, [...] die sogenannten Wessis als Ausbilder. [...] Ich war insofern sehr beeindruckt: Denen war zum Beispiel auch ganz wichtig, dass sie uns mit unserer Mentalität verstehen [...]: »Ich will von euch lernen, und wir wollen uns austauschen.« [...] Also die haben sich, glaub' ich, reingekniet. Haben sogar Wochenenden mit uns verbracht, einfach um uns mal kennenzulernen. Man kann sagen: fast Familie. Das war für mich beeindruckend, das war ein gutes Miteinander. Wir hatten auch viel Spaß mit dem »viertel vor« und »viertel nach«. Ich habe das sehr positiv in Erinnerung. ${ }^{27}$

Auch heute noch, glaube ich, man würde ähnliche Antworten bekommen. [...] Das ist so eine Blaupause auch auf die aktuelle Situation auch. [...] Man sagt zwar immer, Geschichte wiederholt sich nicht, aber [...] manche Verhaltensweisen ändern sich eben nicht. ${ }^{28}$

Ich denke, dass es wichtig ist, ins Gespräch zu kommen. Miteinander reden. Was mir da immer wieder auffällt, wer im Westen gelebt hat und keinerlei Kontakt in die DDR hatte, den hat es nie interessiert, der hatte keine Brüche. Der hat keinen Bruch erlebt, sondern konnte so weiterleben, als wenn nichts passiert wäre, und hier in der DDR ist alles passiert. [...] Jeder, der hier in Görlitz gewesen ist, hat eine Geschichte zu erzählen, wo ein Betrieb

26 Sprecher im Mehrgenerationenhaus Zwönitz (Erzgebirge) am 6. März 2020.

27 Sprecherin in der Thomaskirche Leipzig am 28. Oktober 2019.

28 Sprecher in Stadtbibliothek Görlitz am 10. Oktober 2019. 
zugemacht worden ist, wo die Kinder weggegangen sind, und das spüren wir ja bis heute. Das ist die Nachwende. ${ }^{29}$

Die Wendezeit war für mich verbunden mit dem persönlichen beruflichen Anfang, insofern hab' ich nicht innerhalb einer Struktur eine Veränderung erlebt, sondern ich hab' eigentlich eine Auflösung erlebt. [...] Auf der anderen Seite war das auch eine wunderbar anarchische Zeit, und man hat einfach, weil man ja keinen fragen konnte, der einem gesagt hat, was man machen soll oder wie man es machen soll, da hat man halt einfach gemacht. Manchmal sehnt man sich ein bisschen zurück nach so einer Zeit. Und dann will ich auch nicht immer so dieses Bashing »der West-Mensch, der uns betrogen hat «. Da gab es halt, [...] schwarze Schafe, vielleicht auch viele schwarze Schafe. Aber man wollte auch eine neue Ordnung haben und brauchte halt einfach Fachleute. Es wurde ja beschlossen, dass das eine Land in dem anderen aufgeht, und dann musste man sich eben sozusagen dem unterordnen. ${ }^{30}$

Zuzuhören, verschiedene Perspektiven und Haltungen zuzulassen und zu tolerieren, in einer »coolen« Distanz zur heißen ersten Phase die Transformation und ihre Ausprägungen und Auswüchse zu analysieren und zu bewerten, das wären (als Ergebnis unserer Forschungen) probate Mittel, auf dem Weg der Vereinigung voranzukommen. Die gegenwärtig junge und die mittlere, die »Wende«-Generation haben hierfür die nötige Offenheit und ihr geht der mentale Vereinigungsprozess zu langsam voran.

Ich fand auch den Clip mit den Jugendlichen spannend, weil das tatsächlich dieselben Themen sind, auf die man auch stößt, wenn man heute mit Schülern, Studenten und allen möglichen jüngeren Leuten in der Stadt sich unterhält. [...] Wir haben ja nur die Erfahrungen durch unsere Eltern gemacht. Aber viele Einstellungen, auch dieses Ost-West-Ding, haben sich noch gar nicht so stark verändert. Die sind zum Teil stagniert irgendwie [...], so Klischees, dass die sich jetzt über 30, 40, 50 Jahre eigentlich nicht verändert haben. In der Gesellschaft und wir selbst in unserer Generation [haben] quasi Teile dieser Klischees übernommen [...] und auch so bestimmte Meinungen gelernt $[\ldots] .^{31}$

Ich glaub', es geht extrem langsam voran. Ich hab' halt selbst die Erfahrung gemacht, wenn ich in westdeutschen Bundesländern bin, dass einem viele

29 Sprecher in der Stadtbibliothek Görlitz am 10. Oktober 2019.

30 Sprecher in der Stadtbibliothek Chemnitz am 11. Oktober 2019.

31 Sprecher in der Stadtbibliothek Görlitz am 10. Oktober 2019, Nach-Wende-Generation, Ostdeutschland. 
Fragen gestellt werden. sJa, wie ist denn das bei euch, wie ist denn das und wie ist der Akzent, und wie reden die Leute, und wir sind so ..., und die Arbeitsbedingungen sind so unterschiedlichヶ, und so ganz viele Themen. Gemeingesellschaftlich denkt man, da sind wir schon lange drüber, und die beiden deutschen Staaten gibt es gar nicht mehr, aber irgendwie gibt es sie doch, weil sie in den Köpfen noch existieren. ${ }^{32}$

\section{$5 \quad$ Fazit}

Die Sicherung, Erschließung und Analyse der 40 sächsischen lokalen Fernsehprogramme als audiovisuelles Kulturgut und dessen heutige Wiederaufführung vor Angehörigen der »Vor-Wende-«, der »Wende-« und der »Nach-Wende-Generationen« trägt zur Revision vertrauter Darstellungen, Positionen und Beurteilungen dieser Sattelzeit bei: Dies zeigen erste Programmanalysen und die Auswertungen von Fokusgruppen-Gesprächen. 30 Jahre nach dem Beginn des Vereinigungsprozesses sind ein guter zeitlicher Abstand, aus dem heraus diese drei Generationen sowohl in Ost als auch in West aus kühler Distanz besser verstehen, wie ihre Identität durch das historische Schlüsselereignis des Falls der Berliner Mauer und des Endes des Kalten Krieges verändert beziehungsweise neu geprägt wurde. Und dieser zeitliche Abstand wird der heutigen jüngeren Generation sowie denen nach ihnen helfen nachzuvollziehen und zu verstehen, woher ihre Identitäten kommen und wie sie geformt wurden.

Programmanalysen, regional unterschiedlich gewichtete Präsentationen und Kontextualisierungen der audiovisuellen Quellen entsprechend den oben genannten Kategorien werden das exkarnierte Gedächtnis gegenwärtiger und künftiger Generationen als kulturelles Speichergedächtnis, wenn nicht gar kanonisiertes Funktionsgedächtnis, wesentlich mitgestalten. Weit mehr als schriftliche Quellen, aber auch als Fotografien sind diese bewegten Fernsehbilder und -töne geeignet, Erinnerung und Anschauung eines entscheidenden Abschnitts der jüngsten Zeit-, Sozial- und Kulturgeschichte hervorzurufen, zu reaktivieren, in Lehr- und Bildungspaketen und in Ausstellungen zu erhalten.

Zur Beantwortung der beiden Forschungsfragen:

32 Sprecher in der Stadtbibliothek Görlitz am 10. Oktober 2019, Nach-Wende-Generation, Ostdeutschland. 
1. Inwieweit lassen sich aus der Begegnung mit diesen historischen Programmen Lebenserfahrungen sowie verschüttete beziehungsweise verdrängte Kontexte und Gefühle dieser Sattelzeit bei heutigen Seher*innen re-aktualisieren?

Hier ist zu differenzieren zwischen den drei Generationengruppen. Bei den Zeitzeug*innen wurden (damalige, aber auch gegenwärtige) Zukunfts- und Existenzängste, Traumatisierungen sowie die Gefühle der Selbst- und Fremdscham re-aktualisiert. Ost-WestVorurteile werden erinnert, aber unterschiedlich bewertet. Ein erheblicher Teil dieser Gefühle ist heute noch virulent, unabhängig davon, ob die Zeitzeug*innen eher eine positive oder eher eine negative Bilanz der Vereinigung für sich ziehen. Für die beiden jüngeren Generationen können die Autor*innen wegen deren relativ geringen Anteils an den Fokusgruppen nur vorläufig festhalten: Ängste und Traumatisierungen sind hier nicht anzutreffen; stattdessen ein begieriges, dankbares Aufsaugen von audiovisuell induzierten Anschauungen des damaligen Alltags, der ohne die Fernsehbeiträge aus dem Nahbereich allenfalls aus Kindeserinnerungen, Erzählungen oder erlebten Erziehungsmaßstäben der Eltern sowie aus Schule und Hochschule bestand.

2. Inwieweit können dadurch Erklärungen für die Wurzeln gegenwärtiger sozialer und politischer Diskurse im vereinigten Deutschland gegeben werden?

Bis heute treffen bei Ost- und Westdeutschen zwei unterschiedliche kulturelle, soziale Arbeits- und Erfahrungswelten aufeinander. Aufgrund des gemeinsamen Zeitraums von immerhin drei Jahrzehnten existiert die Polarisierung »Ost versus West« nicht mehr in der Reinform wie noch während der Sattelzeit. Vielerlei Differenzierungen, Mischungen und gemeinsame Erfahrungen sind eingetreten. Doch erhoffte und erfahrene Veränderungen waren nicht immer kongruent, und trotz beiderseitigen Bemühens: Die »Große mentale Transformation « - und damit die Vollendung der Vereinigung - ist ein bis heute und darüber hinaus anhaltender, unvollendeter Prozess. 


\section{Literatur}

Albrecht, Gerd. 1969. Nationalsozialistische Filmpolitik. Eine soziologische Untersuchung über die Spielfilme des Dritten Reichs. Stuttgart: Enke.

Antennengemeinschaft Chemnitz/Ebersdorf. Chronik. http://www.antenneebersdorf.de/index.php/geschichte.html. Zugegriffen: 17. März 2021.

Antenne Meuselwitz (Sendestart 1986). http://www.antenne-meuselwitz.de/ gesch.htm. Zugegriffen: 17. März 2021.

Assmann, Aleida. 2014. Geschichte im Gedächtnis. Von der individuellen Erfahrung zur öffentlichen Inszenierung. München: C. H. Beck.

Assmann, Aleida. 2018. Der lange Schatten der Vergangenheit. Erinnerungskultur und Geschichtspolitik. München: C.H. Beck.

Buchholz, Arnold. 1968. Die große Transformation. Stuttgart: Deutsche Verlags-Anstalt.

ARD. o.J. Chronik der ARD 1978. http://web.ard.de/ard-chronik/index/ 2190? year=1978. Zugegriffen: 17. März 2021.

Eckert, Rainer. 2007. Gegen die Wende-Demagogie - für den Revolutionsbegriff. Anmerkungen zu: Michael Richter, Die Wende. Plädoyer für eine umgangssprachliche Benutzung des Begriffs. Deutschland Archiv 6: 1084-1086.

Einigungsvertrag. 1990. Vertrag zwischen der Bundesrepublik Deutschland und der Deutschen Demokratischen Republik über die Herstellung der Einheit Deutschlands. EinigVtr. Ausfertigungsdatum: 31.08.1990. https://www.gesetze-im-internet. de/einigvtr/EinigVtr.pdf. Zugegriffen: 17. März 2021.

Erll, Astrid. 2017. Kollektives Gedächtnis und Erinnerungskulturen. Stuttgart: J.B. Metzler. DOI: 10.1007/978-3-476-05495-1_1.

Gerhards, Jürgen. 1994. Politische Öffentlichkeit. Ein system- und akteurstheoretischer Bestimmungsversuch. Kölner Zeitschrift für Soziologie und Sozialpsychologie, Sonderheft Öffentlichkeit und soziale Bewegungen: 77-105.

Großgemeinschaftsantennenanlage Marienberg-Goldkindstein. 202040 Jahre Antennengemeinschaft Marienberg-Goldkindstein. Interview mit Günter Rötzer. MittelErzgebirgsFernsehen (MEF) vom 1. Februar, https://www.youtube.com/ watch? $v=$ mkcSNbOSZLk. Zugegriffen: 17. März 2021.

Hensel, Jana. 2017 [2002]. Zonenkinder. Reinbek bei Hamburg: Rowohlt.

Hofmann, Michael. 2016. Einführung. In: Helden im Wilden Osten. Ostdeutschland in den Neunzigerjahren. Begleitheft zur Ausstellung, herausgegeben von Christian Schmidt und Aiko Wulf. Leipzig.

Jesse, Eckhard. o. J. Der Begriff »Wende« in der Diskussion. Erfurt: Gesellschaft für Zeitgeschichte e.V. http://www.gesellschaft-zeitgeschichte.de/dokumente/aktuelle-dokumente/der-begriff-wende/. Zugegriffen: 17. März 2021.

Koselleck, Reinhart. 1972. Einleitung. In: Geschichtliche Grundbegriffe. Historisches Lexikon zur politisch-sozialen Sprache in Deutschland, herausgegeben von Otto Brunner, Werner Conze und Reinhart Koselleck, XIII-XXVII. Stuttgart: KlettCotta.

Kowalczuk, Ilko-Sascha. 2019. Die Übernahme. Wie Ostdeutschland Teil der Bundesrepublik wurde. München: C.H. Beck. 
Kretzschmar, Judith und Rüdiger Steinmetz. 2020. „Wir hatten immer Sendezeit, wie wir wollten.« Rundfunkhistorisches Gespräch mit Gerd Simmank (Laubuscher Heimatkanal). Rundfunk und Geschichte 46 (1-2): 86-101.

Kühn, Detlef. 2003. Den Privaten eine Anstalt: Gründung und erste Jahre der SLM. In: Rundfunk in Ostdeutschland. Erinnerungen - Analysen - Meinungen, herausgegeben von Gerlinde Frey-Vor und Rüdiger Steinmetz, 91-107. Konstanz: UVK.

Lamnek, Siegfried. 1995. Qualitative Sozialforschung. 2 Bände. Weinheim: Beltz PVU.

Lamnek, Siegfried. 1998. Gruppendiskussion. Theorie und Praxis. Weinheim: Beltz PVU.

Lamnek, Siegfried, und Claudia Krell. 2016. Qualitative Sozialforschung. Weinheim: Beltz PVU.

Milev, Yana. 2020. Das Treuhand-Trauma: Die Spätfolgen der Übernahme. Berlin: Das neue Berlin.

McCreary, Eugene C. 1972. Zeitgeschichte im Film- und Tondokument: 17 historische, pädagogische und sozialwissenschaftliche Beiträge, herausgegeben von Günter Moltmann und Karl Friedrich Reimers. 1970. Göttingen: Musterschmidt. Rezension in The American Historical Review 77(2): 473-474. DOI: https://doi. org/10.1086/ahr/77.2.473-a.

Neubert, Erhart. 2008. Unsere Revolution. Die Geschichte der Jahre 1989/90. München, Zürich: Piper.

Pasternack, Peer. 1998. »Demokratische Erneuerung«. Eine universitätsgeschichtliche Untersuchung des ostdeutschen Hochschulumbaus 1989-1995. Mit zwei Fallstudien: Universität Leipzig und Humboldt-Universität zu Berlin. Dissertation. Carl von Ossietzky-Universität Oldenburg. https://www.hof.uni-halle.de/web/dateien/Demokratische-Erneuerung.pdf. Zugegriffen: 17. März 2021.

Polanyi, Karl. 2019 [1944]. The Great Transformation. Politische und ökonomische Ursprünge von Gesellschaften und Wirtschaftssystemen. Frankfurt am Main: Suhrkamp.

Richter, Michael. 2007a. Die Wende. Plädoyer für eine umgangssprachliche Benutzung des Begriffs. Deutschland Archiv 5: 861-868.

Richter, Michael. 2007b. Replik auf Rainer Eckerts Anmerkungen. Deutschland Archiv 6: 1086-1087.

Rötzer, Günter. 1984. An den Rat des Kreises Marienberg (Erzgebirge): »Kabelrundfunk im Gebiet Marienberg«, 3. August 1984. https://www.mef-line.de/ über-uns/entwicklung.html. Zugegriffen: 17. März 2021.

Schröder, Richard. 2007. Die wichtigsten Irrtümer über die deutsche Einheit. Freiburg im Breisgau: Herder.

Schweinitz, Jörg, Hilde Hoffmann und Tobias Ebbrecht. 2009. Zur Einleitung. Die Erinnerung an die DDR, der Dokumentarfilm und die Filmgeschichtsschreibung. In: DDR erinnern vergessen. Das visuelle Gedächtnis des Dokumentarfilms, herausgegeben von Jörg Schweinitz, Hilde Hoffmann und Tobias Ebbrecht, 7-19. Marburg: Schüren.

Staud, Toralf. 1996. Fernsehen von nebenan. Die Zeit Nr. 34 vom 16. August.

Steinmetz, Rüdiger, und Reinhold Viehoff (Hrsg.). 2008. Deutsches Fernsehen Ost. 
Eine Programmgeschichte des DDR-Fernsehens. Potsdam: Verlag für Berlin-Brandenburg.

Ther, Phillipp. 2016. Die neue Ordnung auf dem alten Kontinent. Eine Geschichte des neoliberalen Europa. Berlin: Suhrkamp.

Ther, Phillipp. 2019. Das andere Ende der Geschichte. Über die Große Transformation. Berlin: Suhrkamp.

Weber, Petra. 2020. Getrennt und doch vereint. Deutsch-deutsche Geschichte 19451989/90. Berlin: Metropol.

Wolfrum, Edgar. 2006. Die geglückte Demokratie. Geschichte der Bundesrepublik Deutschland von ihren Anfängen bis zur Gegenwart. Stuttgart: Klett-Cotta.

Wollmann, Hellmut. 1997. Transformation der politisch-administrativen Strukturen in Ostdeutschland. Wiesbaden: Springer VS.

\section{Open Access}

Dieser Beitrag erscheint unter der Creative-Commons-Lizenz CC BY-ND 3.0 DE: https://creativecommons.org/licenses/by-nd/3.0/de/. 